\title{
Severe aortic valve fusion after nearly three years of support with the Novacor left ventricular assist system
}

\author{
Motonobu Nishimura, MD, Shegeaki Ohtake, MD, Yoshiki Sawa, MD, Norihide Fukushima, MD, Goro Matsumiya, MD, \\ and Hikaru Matsuda, MD, PhD, Osaka, Japan
}

$\mathrm{P}$

artial aortic valve fusion induced by a left ventricular assist system (LVAS) has been reported by Rose and associates. ${ }^{1}$ We report here a case of fish mouth-shaped aortic valve fusion induced by prolonged LVAS circulatory support.

\section{Clinical Summary}

A 48-year-old man first had congestive heart failure in 1994, and medical treatment was initiated. In 1995, the heart failure worsened despite medication, and the patient required hospitalization and intensive treatment. Full examination of the patient led to the diagnosis of idiopathic cardiomyopathy with an enlarged left ventricle and depressed contractile function. Endomyocardial biopsy showed findings compatible with idiopathic cardiomyopathy. The patient's heart failure improved with intensive treatment, and he was discharged with enhanced medical therapy. In September 1997, the patient's heart failure symptoms again gradually worsened, and in December of that year he was brought to the emergency department with cardiogenic shock. He was intubated, placed on intra-aortic balloon pump support, and given a regimen of the maximum dose of catecholamine, but his condition did not improve despite this aggressive medical treatment. At that point no aortic valve disease had been pointed out by either cardiac catheterization or echocardiography. In March 1998 the patient was transferred to Osaka University Hospital and underwent the placement of a Novacor LVAS (World Heart Inc, Oakland, Calif). In June 1998 the patient was qualified as a candidate for cardiac transplantation by the institutional review board and the heart transplantation committee of the Japanese Circulation Society, and he was then registered with the Japan Organ Transplantation Network. Our intention was to treat this as a bridge to transplantation, although the legislation for organ transplantation is extremely strict in Japan,, 3 and the operational mode for the Novacor LVAS was essentially set to fill-to-empty mode, which had been keeping his aortic valve closed.

Because he was the first patient in Japan ever to be discharged with LVAS support to await heart transplantation, the patient

From the Division of Cardiovascular Surgery, Department of Surgery, Osaka University Graduate School of Medicine, Osaka, Japan.

Novacor LVAS, World Heart Inc, Oakland, Calif.

Received for publication Dec 11, 2001; accepted for publication Dec 15, 2001.

Address for reprints: Hikaru Matsuda, MD, PhD, Department of Surgery (E1), Osaka University Graduate School of Medicine, 2-2 Yamada-oka, Suita, Osaka 565-0871, Japan (E-mail: matsuda@surg1.med.osakau.ac.jp).

J Thorac Cardiovasc Surg 2002;124:179-80

Copyright $\odot 2002$ by The American Association for Thoracic Surgery

$0022-5223 / 2002 \$ 35.00+0 \quad \mathbf{1 2 / 5 4 / 1 2 2 3 4 9}$

doi: $10.1067 / \mathrm{mtc} .2002 .122349$

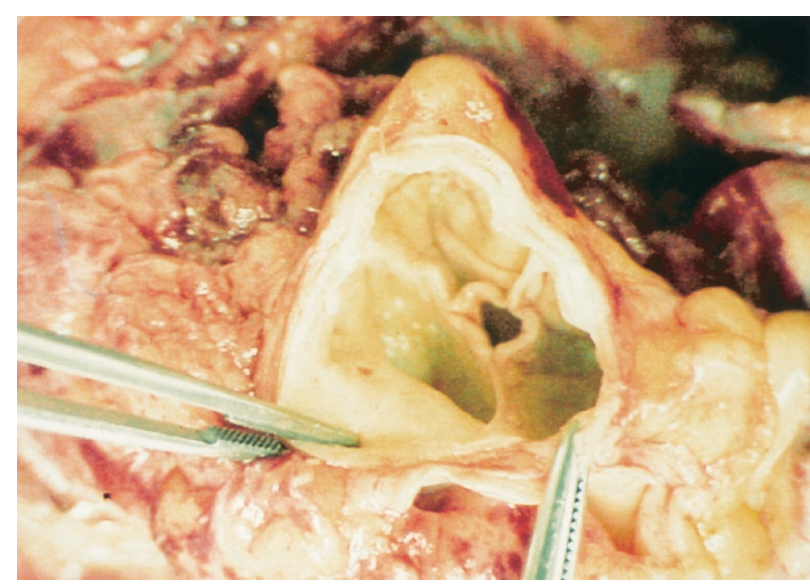

Figure 1. Fused aortic valve in explanted heart.

repeated short leaves for 4 months and finally was discharged home 7 months after LVAS implantation. While awaiting heart transplantation, the patient enjoyed excellent quality of life at home, except for a short hospital admission for a minor infection in the drive line exit site. Serial echocardiography revealed no functional recovery of his native heart. The anticoagulation regimen included coumadin and aspirin, and the patient's condition was fairly stable with a regimen of 3.5 to $4 \mathrm{mg}$ coumadin, with an international normalized ratio of 2.5 to 3.5. During the waiting for heart transplantation, the patient did not have any thromboembolic episodes. On February 26, 2001, after a wait on LVAS support for 1087 days, a brain-dead donor of identical blood type became available and the patient underwent orthotopic cardiac transplantation. The patient had hemodynamically compromised vascular rejection, which was successfully treated with plasma exchange, and he was discharged home on postoperative day 75 in good physical and mental condition. After nearly 3 years, no degeneration was found in both inflow and outflow valves of the explanted Novacor LVAS. Smooth and rigid peel formation was found inside the inflow conduit. The explanted heart showed macroscopically diffuse fibrosis in both ventricles and the intraventricular septum. Interestingly, the aortic valve was fused between commissures and only small opening was left (Figure 1). Histologic examination revealed fibrous fusion between the commissures.

\section{Discussion}

Rose and associates ${ }^{1}$ reported partial aortic valve fusion induced by LVAS. They found partial fusion of one or two aortic commissures in 4 of 6 patients. After nearly 3 years of LVAS support in automatic fill-to-empty mode, our patient's aortic valve was fused in all the commissures and had the shape of a fish mouth, although 
no thrombi were found around the aortic valve. As far as we are aware, there have been no previous reports describing the aortic valve fusion of all three commissures, although Myers and colleagues ${ }^{4}$ reported complete fusion of a bioprosthetic valve in the aortic position during several days of support with left heart bypass. This complication may become serious for the patients in whom LVAS acts as a bridge to recovery or in those in whom LVAS fails. Fortunately, neither was the case in our patient, but this complication should be kept in mind for the patient supported by LVAS for an extended period. Even though our patient was receiving the rather strict and stable anticoagulant therapy recommended for the Novacor LVAS, commissural fusion occurred. This phenomenon may occur more easily with the HeartMate VE LVAS (Thoratec Corporation, Pleasanton, Calif) because that device requires less anticoagulant therapy. Considering mechanical failure of LVAS, switching to the low-rate fixed mode for several minutes at least once a day may be effective to prevent aortic valve fusion of the native heart.

\section{References}

1. Myers JL, Bull A, Kastl DG, Pierce WS. Fusion of prosthetic valve during left heart bypass. J Thorac Cardiovasc Surg. 1981;82:263-7.

2. Matsuda H, Fukushima N, Sawa Y, Nishimura M, Matsumiya G, Shirakura R. First brain dead donor heart transplantation under new legislation in Japan. Jpn J Thorac Cardiovasc Surg. 1999;47:499-505.

3. Nishimura M, Ohtake S, Sawa Y, Fukushima N, Yamaguchi T, Matsuda H. Left ventricular assist systems as a bridge to heart transplantation: the role and the strategy in Japan. Transplant Proc. 1999; 31:1997-9.

4. Rose AG, Park SJ, Bank AJ, Miller LW. Partial aortic valve fusion induced by left ventricular assist device. Ann Thorac Surg. 2000;70: $1270-4$. 